\title{
PHOTOMETRIC FACILITIES OF THE AIST SPACE PROJECT
}

\author{
I.M.KOPYLOV, D.L.GORSHANOV AND M.S.CHUBEY \\ Pulkovo Observatory,196140 St Petersburg, Russia \\ e-mail: mchubey@gaoran.spb.su
}

\begin{abstract}
The arguments are given of the choice for the Vilnius seven color medium band photometric system as the most effective one for the AIST space project. A prediction is made of the limiting stellar magnitudes $V$ for every Vilnius passband and the integral band $T$ at $\sigma_{\Sigma} \leq 30 \mathrm{mmag}$ from a 3 year $A I S T$ mission.
\end{abstract}

\section{Introduction}

The main aim of the scientific program of the AIST project is to create a fundamental catalogue of positions, proper motions and parallaxes for faint stars at the epoch of mission with milliarcsecond precision, to carry out their high precision multicolor photometry and direct optical measurements $\mathrm{A}$ the precise quasar coordinates to fit a fundamental system. The last was the main reason for a choice of the limiting magnitudes to be of the order of $V_{\text {lim }}=18 \mathrm{mag}$.

\section{Instrumentation}

We estimate for a two Schmidt telescopes instrument. The version is: one of two telescopes has a beam combiner, another one, with full pupil, has no beam combiner. As the standard detector units we use (Fig.1) a square CCD chips with $256 \times 256$ pixels $(4.1 \times 4.1 \mathrm{~mm})$, the pixels being $16 \mu \mathrm{m}=1.32^{\prime \prime}$ squares. The central diffraction spot for the full telescope pupil is $d_{0}=$ $0.75^{\prime \prime}$. The adopted spectral sensitivity $Q E(\lambda)$ of CCD has values $56,76,77$ and $42 \%$ for the wavelength $300,500,700$ and $900 \mathrm{~nm}$ respectively. At the angular velocity of $150^{\prime \prime} / \mathrm{s}$ the star crosses the field of view within $34^{s}$ and the single CCD chip within $2.25^{s}$. The main axis of rotation has a scan step $20^{\prime}$ and small drift during one rotational period. 


\section{The Choice of the Photometric System}

We have compared the parameters and qualities of several modern most usable multicolor photometric systems. The criteria have been used: (a) the of the selected passbands and their positions, fixing spectral type and luminosity, and domains of application of the system; (b) the bandwidths and their comparative abilities in achieving the necessary limiting magnitude of the whole system; (c) the system purity parameters (Jaschek and Frankel, 1986), i.e. the quality and ability of the photometric system to classify stars with different characteristics in the optimal way and to reveal and classify the various types of peculiar stars, for example, Ap, Am, carbon and metal-poor stars, subdwarfs, white dwarfs, some types of binaries etc.; (d) the availability of a sufficient number of standards, including both ordinary and peculiar stars observed in a given photometric system; (e) the possibility of transformation of the photometric data collected in the chosen system to other systems with a minimum loss of precision. These criteria were applied for the comparison of the $U B V R I$, uvby $+\beta_{n}+\beta_{w}$, Vilnius and Geneva photometric systems. The firm conclusion has been reached that the most effective one is the medium-band seven-color Vilnius system WPXYZVS (Straižys 1977 and 1992).

After that, we compared main merits and problems of the basic versions of this system: Standard, VilGen, Interferometric and CCD-adapted (Straižys 1977; Straižys et al. 1992). As a final result, we have used the Interferometric version (Int) whose parameters are listed in Table 1. The contents of columns 1 - 4 are understandable. The 5 th column contains the integrated filter transparency (the equivalent width $W_{\lambda}$ ).

\section{Calculation Scheme}

For the calculation of the expected limiting magnitudes $V_{\text {lim }}$, the magnitude error in $1^{s}$ exposure $\sigma_{1}$ and the error in the whole flight time $\sigma_{\Sigma}$ we use the standard formula

$$
\sigma=\frac{\sqrt{N_{*}+N_{n}+N_{C C D}}}{N_{*} \sqrt{t}}
$$

Energy distribution $E\left(\lambda_{i}\right)$ in the spectrum of the faint stars, of the faint stars background $(S L)$ and of Zodiacal light $(Z L)$ is similar to that of a star of spectral type $G$. With the adopted aperture, optics transparency $p\left(\lambda_{i}\right), W\left(\lambda_{i}\right)$ and $Q E\left(\lambda_{i}\right)$ we find for every band the value $N_{*}$ in units $\left[e^{-} / s\right]$. For the calculation of $N_{n}$ in formula (1) we have used the mean integrated sky brightness $\overline{m_{i}}(Z L+S L)$ per pixel given in column 8 of Table 1 .

All the calculation for the band $T$ (the last row of Table 1 ) was made taking into account only the losses of light in optics and the $Q E(\lambda)$ of the 
TABLE 1. The adopted version of the Vilnius system.

\begin{tabular}{|c|c|c|c|c|c|c|c|c|}
\hline Band & $\begin{array}{c}\lambda_{0} \\
\mathrm{~nm}\end{array}$ & $\begin{array}{l}\Delta \lambda \\
\mathrm{nm}\end{array}$ & $\begin{array}{c}\tau_{\max } \\
\%\end{array}$ & $\begin{array}{l}W_{\lambda} \\
\mathrm{nm}\end{array}$ & $\frac{W_{\lambda}(I n t)}{W_{\lambda}(S t d)}$ & $\begin{array}{c}\text { QE } \\
\%\end{array}$ & $\begin{array}{c}\overline{\frac{m_{i}}{p i x}} \\
m a g\end{array}$ & $\begin{array}{c}t_{\text {total }} \\
\mathrm{s}\end{array}$ \\
\hline 1 & 2 & 3 & 4 & 5 & 6 & 7 & 8 & 9 \\
\hline W & 350 & 52 & 65 & 35.5 & 2.10 & 62 & 21.7 & 670 \\
\hline $\mathrm{P}$ & 375 & 20 & 58 & 12.2 & 1.11 & 65 & 21.7 & 1080 \\
\hline $\mathrm{X}$ & 406 & 17 & 60 & 10.7 & 1.57 & 68 & 21.6 & 1005 \\
\hline$Y$ & 468 & 23 & 82 & 19.8 & 2.61 & 74 & 21.4 & 310 \\
\hline $\mathrm{Z}$ & 518 & 18 & 74 & 14.0 & 1.47 & 77 & 21.1 & 380 \\
\hline V & 547 & 26 & 85 & 23.2 & 3.14 & 79 & 20.9 & 285 \\
\hline $\mathrm{S}$ & 656 & 32 & 85 & 28.6 & 7.94 & 80 & 20.5 & 205 \\
\hline $\mathrm{T}$ & 625 & 587 & 100 & 548 & - & 80 & 20.2 & 105 \\
\hline
\end{tabular}

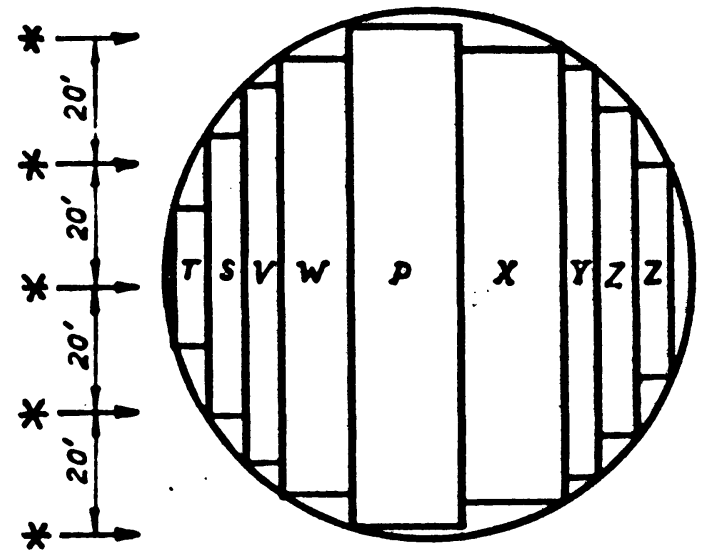

Figure 1. Relative areas in the field of view covered by 7 Vilnius filters (and by T-band), to reach the same $V_{l i m}$ magnitude of a G2 IV-type star.

CCD. The total noise $N_{C C D}$ in the formula (1) was assumed to be $5 e^{-} s^{-1}$. A "drift-scan" principle was used throughout calculations.

\section{Analysis of the Results}

Extensive tables were prepared, containing the relations between $V$ and $\sigma_{1}$ for the bands of the Vilnius system and for the $T$-band. These tables demonstrate that because of the rather small $W_{\lambda}$ values for the $P$ and $X$ bands and relatively low $E\left(\lambda_{i}\right)$ for the $W$ band, the magnitudes $V$ (or $V_{\text {lim }}$ ) at some adopted $\sigma$ (or $\sigma_{\text {lim }}$ ) for these bands are smaller than $\bar{V}$ (or $\bar{V}_{\text {lim }}$ ) of the remaining four Vilnius bands by $1.6,1.3$ and $0.8 \mathrm{mag}$ respectively. The best possibility for an approximate equalizing of $V_{l i m}$ for different bands is 
to enlarge the relative widths and lengths of the chips within the field of view covered by the "poor" filters $P, X$ and $W$.

The result of one such numerical equalization of $V_{\text {lim }}$ (the number and size of CCD chips covered by the Vilnius filters) is presented in Fig. 1.

We have estimated also the approximate scanning times (column 9 of Table 1) for the Vilnius bands during the time of the AIST mission (3 years) which would be necessary in order to reach nearly the same $V_{i}$.

In Table 2 we represent the dependences of the final errors for all the Vilnius and $T$ bands. The upper row of asterisks marks $\sigma_{\Sigma} \leq 1 \mathrm{mmag}$, at $V \approx 11 \mathrm{mag}$, the lower one shows us the $V_{\text {lim }}$ at $\sigma_{\Sigma} \leq 30 \mathrm{mmag}$.

TABLE 2. The final errors $\sigma_{\Sigma}$ of the predicted stellar magnitudes $V_{i}$ of G2IV-star. Total flight time is 3 years. Errors are given in $\mathrm{mmag}$.

\begin{tabular}{ccccccccc}
\hline$V$ & $\sigma_{W}$ & $\sigma_{P}$ & $\sigma_{X}$ & $\sigma_{Y}$ & $\sigma_{Z}$ & $\sigma_{V}$ & $\sigma_{S}$ & $\sigma_{T}$ \\
\hline 11 & $*_{0.92 *}$ & $*_{1.07 *}$ & $*_{0.94 *}$ & ${ }^{*} 0.92^{*}$ & $*_{1} 1.00^{*}$ & ${ }^{*} 0.98^{*}$ & $*_{0.92 *}$ & 0.32 \\
12 & 1.42 & 1.71 & 1.51 & 1.60 & 1.56 & 1.40 & 1.46 & 0.51 \\
13 & 2.3 & 2.8 & 2.4 & 2.4 & 2.4 & 2.2 & 2.3 & ${ }^{*} 0.80^{*}$ \\
14 & 3.7 & 4.5 & 3.8 & 4.0 & 4.0 & 3.5 & 3.7 & 1.28 \\
15 & 6.1 & 7.5 & 6.4 & 6.4 & 6.4 & 5.7 & 5.9 & 2.0 \\
16 & 10.4 & 13.3 & 10.8 & 10.5 & 10.6 & 9.2 & 9.6 & 3.2 \\
17 & 18 & $* 26 *$ & 21 & 18 & 18 & 15.5 & 16 & 5.2 \\
18 & $* 37 *$ & 55 & $* 42 *$ & $* 32 *$ & $* 35 *$ & $* 28 *$ & $* 29 *$ & 8.6 \\
19 & 77 & 125 & 99 & 70 & 72 & 58 & 56 & 15 \\
20 & 178 & & 230 & 147 & 107 & 119 & 118 & $* 28 *$ \\
\hline
\end{tabular}

\section{Conclusion}

The calculation shows that the $A I S T$ project would obtain medium band multicolor photometry of faint stars down to $18.0 \mathrm{mag}$ with a final error $\sigma_{\Sigma} \leq 30 \mathrm{mmag}$, photometry of bright stars down to $\approx 11 \mathrm{mag}$ with $\sigma_{\Sigma} \leq$ $1 \mathrm{mmag}$ and surface photometry of extended objects down to $\approx 18 \mathrm{mag}$ per pixel with $\sigma_{\Sigma} \leq 30 \mathrm{mmag}$. In addition, the $T$ band allows to measure stars with the same precision but $2.0-2.5 \mathrm{mag}$ fainter.

\section{References}

Jaschek C., Frankel S., 1986, A\&Ap, 158, 174.

Straižys V., 1977, Multicolor Stellar Photometry, Mokslas, Vilnius.

Straižys V., 1992, Baltic Astronomy, 1, 107.

Straižys V., Boyle R.P., Kuriliene G., 1992, Baltic Astronomy, 1, 95. 